\title{
Keine kardiovaskuläre Demenzprävention?
}

\author{
Kann die Entwicklung einer Demenz durch eine ambulante Kontrolle und Korrektur multipler kardio- \\ vaskulärer Risikofaktoren verhindert werden? Eine große prospektive Studie gibt Anlass zur Skepsis.
}

_ Für die Studie wurden 3.526 Teilnehmer im Alter von 70-78 Jahren in 116 Allgemeinarztpraxen rekrutiert. 1.636 von ihnen wurden wie gewohnt behandelt. 1.890 hingegen konsultierten zur Erfassung kardiovaskulärer Risikofaktoren alle vier Monate eine Pflegeperson in der Praxis. Alle zwei Jahre wurde Blut entnommen. Auf dieser Basis wurden individuelle Gesundheitsanweisungen mit motivierenden Gesprächstechniken vermittelt. Hypertonus, Dyslipidämie und Typ-2-Diabetes wurden nach Bedarf medikamentös behandelt.

Nach durchschnittlich 6,7 Jahren hatten in beiden Gruppen 7\% der Teilnehmer eine Demenz entwickelt. Auch das Ausmaß körperlicher Behinderungen war gleich. In beiden Gruppen waren 16\% der Teilnehmer im Beobachtungszeitraum verstorben.

- Madjd A et al. Beneficial effect of high energy intake at lunch rather than dinner on weight loss in healthy obese women in a weight-loss program: a randomized trial. Am J Clin Nutr. 2016;104:982-9

\section{KOMMENTAR}

Schon Alois Alzheimer hat auf amyloidartige Veränderungen an zerebralen Arteriolen und Arterien hingewiesen. Dies hat vermutlich klinische Relevanz: Bei vielen Alzheimer-Patienten mit typischem Krankheitsverlauf finden sich im MRT neben allgemeiner Atrophie auch vaskuläre Marklagerläsionen. Die hierzu wahrscheinlich beitragenden kardiovaskulären Risikofaktoren sind eigentlich auch beeinflussbar.

Angesichts mangelnder Fortschritte in der kausalen Behandlung der AmyloidPlaque-Pathologie ist der präventive Ansatz in einem allgemeinmedizinischen Setting eigentlich wirklich gut. Auch die vorliegende Studie aus Amsterdam ist sehr gut gemacht. Dennoch zeigte sich kein Effekt. Die Erklärung ist eigentlich naheliegend: Die Teilnehmer waren zu alt, und die Therapie setzte zu spät ein. Sollte sich ein Effekt mit einer Prävention beim Vorliegen von Risikofaktoren erzie-

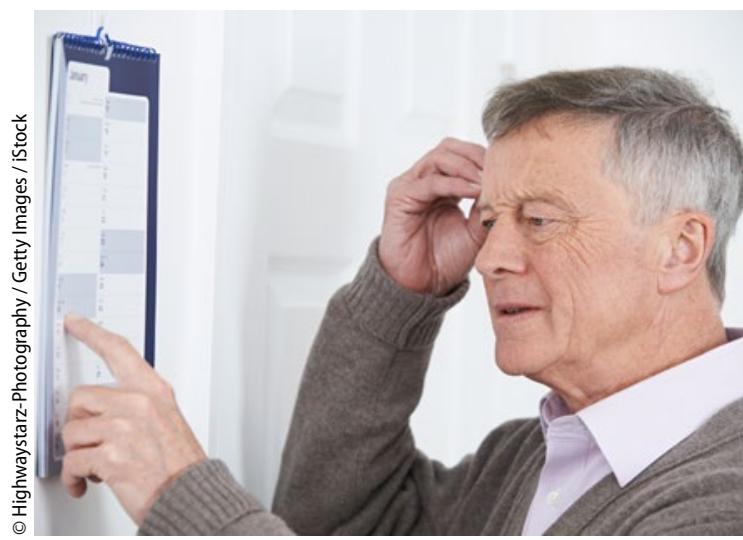

Für eine Intervention ist es zu spät.

len lassen, müsste diese wohl viel früher beginnen und länger anhalten. Dies können Studien vermutlich niemals abbilden. Wir Ärzte können also nur dazu raten, die beeinflussbaren Risikofaktoren schon im mittleren Lebensalter zu kontrollieren und zu behandeln.

Prof. Dr. med. M. Weih

\section{Hier steht eine Anzeige.}

\author{
Springer
}

